\title{
Primary Localized Amyloidosis of the Intestine: A Pathologist Viewpoint
}

\author{
Saeed Ali Alshehria, c, Mahmoud Rezk Abdelwahed Hussein ${ }^{\mathrm{b}}$
}

\begin{abstract}
Background: Localized amyloidosis of the intestine is a rare entity, which can clinically masquerade several conditions such as colitis, polyps, and malignant tumors. This study aims to evaluate the clinicopathological features of this entity.

Methods: To evaluate the clinicopathological features of this entity, a comprehensive search of the literature (1960 to 2019) was done using the following keywords: "amyloidosis" and "small intestine" or "duodenum" or "ileum" or "jejunum" or "colon". We identified 756 studies about gastrointestinal amyloidosis. Data were examined for 27 studies about localized intestinal amyloidosis. The clinicopathological features were described.
\end{abstract}

Results: The age at presentation ranged from 29 to 88 years. The male to female ratio was $3: 1$. The jejunum and sigmoid colon were the most commonly involved sites. Abdominal pain and intestinal obstruction (small intestine), or rectal bleeding (sigmoid region) were the most common clinical presentations. Colonoscopic findings included wall thickening, mucosal ulcerations (small intestine), and tumor-like masses (colon).

Conclusions: The clinical presentations of localized intestinal amyloidosis depend on the site of the deposition of the amyloid. In most cases, amyloid deposits consisted of light chain protein.

Keywords: Amyloidosis; Amyloid; Colon; Small intestine; Rectum; Gastrointestinal tract

\section{Introduction}

The term "amyloid" was initially coined to describe materials

Manuscript submitted June 5, 2020, accepted July 10, 2020

Published online August 14, 2020

aDepartment of Pathology, Armed Forces Hospitals, Southern Region, King Fahd Hospital, Saudi Arabia

bDepartment of Pathology, Faculty of Medicine, Assuit University Hospitals, Assuit, Egypt

${ }^{\mathrm{c} C o r r e s p o n d i n g ~ A u t h o r: ~ S a e e d ~ A l i ~ A l s h e h r i, ~ D e p a r t m e n t ~ o f ~ P a t h o l o g y, ~ C h e m-~}$ istry Section, Armed Forces Hospital Southern Region, Khamis Mushyte, Saudi Arabia. Email: syd46118@gmail.com

doi: https://doi.org/10.14740/gr1303 found in plants and has been thought to represent carbohydrate (Greek "amylon" = starch). In medical literature, the term "Amyloidosis" has been known for more than 160 years. It was named after the deposition of the hallmark protein called cross- $\beta$-sheet amyloid fibrils, which represents either misfolded or misassembled proteins. The nomenclature of the amyloid protein uses two letters. The first letter "A" refers to amyloid, whereas the second refers to the precursor proteins [1]. The amyloid proteins include several types such as immunoglobulin light chains (AL) in primary amyloidosis, transthyretin (TTR), beta-2-microglobulin in hemodialysis-associated amyloidosis, and serum amyloid A (SAA) protein in secondary (AA) amyloidosis [2-4]. There are some associations between AL amyloid deposition and several hematological conditions including myeloma, lymphoplasmacytic disorders, and plasma cell dyscrasia. Moreover, AL amyloidosis and myeloma may show similar genetic abnormalities such as $14 \mathrm{q}$ translocations and $13 \mathrm{q}$ deletion [5].

Amyloidoses are degenerative conditions associated with significant suffering not only for patients but also for their families as well [3]. They are characterized by the deposition of extracellular protein fibrils in the organs such as the kidneys, nerves, gastrointestinal tract, heart, and skin. Amyloidoses are associated with significant morbidities such as malabsorption, renal insufficiency, and hematological disorders, or even lifethreatening events such as intestinal obstruction, renal failure, and fatal arrhythmia. The clinicopathological features of amyloidosis are nonspecific. They depend on the type of the precursor protein, site, and severity of the amyloid deposition. The diagnosis of amyloidosis depends on the examination of the tissue biopsies from the lesions. It is established by the detection of amyloid deposits with its characteristic birefringence under polarized light following staining with Congo red, or thioflavin$\mathrm{T}$, or immunohistochemical stains. Management of amyloidosis includes reduction of the supply of amyloid fibril precursor proteins, stabilization of the precursor protein formation, administration of anti-plasma therapies, general care (systemic amyloidosis) or surgical resection (in localized amyloidosis) $[6,7]$.

Amyloidosis includes both primary and secondary types. In the former (also known as AL for amyloid light chain), there is no evidence of coexisting disease and it has an immunoglobulin light chain as a major protein component. In secondary amyloidosis (also known as AA for amyloid-associated), there is evidence of coexisting disease resulting in the production of inherently amyloidogenic proteins or overproduction of potentially amyloidogenic normal proteins, and it has serum amyloid-associated protein (SAA). The deposition of amy- 
loid may be either widespread (systemic amyloidosis) [2, 7, 8] or restricted to a specific organ (localized amyloidosis). In systemic amyloidosis, the amyloidogenic proteins are synthesized at sites far away from the sites of amyloid deposition. The amyloid protein is deposited at several sites and therefore the patients have disparate clinical manifestations such as arrhythmia, neuropathies, malabsorption, and proteinuria due to cardiac, neural, intestinal, and renal involvement, respectively [9]. Currently, the classification of amyloidosis is based on the type of precursor proteins [10].

Localized amyloidosis refers to the infiltration of the tissues by amyloid where the precursor proteins are synthesized at or close to the site of deposition. It includes both organ-specific amyloidoses where the amyloid is produced by the organ itself and non-organ specific amyloidosis where the fibrillar protein is not specific to the affected organ [2]. Localized amyloidosis of the intestine is a rare condition that involves the duodenum, jejunum, and ileum, appendix, or colorectum. The clinicopathological features are nonspecific and therefore they can masquerade several conditions such as collagenous colitis, ischemic colitis, bowel infarction, perforation, polyps, and malignant tumors [11-13]. The colonoscopic features include mucosal granularity, friability, erosions, ulcerations, petechial hemorrhage, and polypoid lesions [14]. The radiological findings include ulcerations, thickening of the mucosal folds, narrowing of the colonic lumen, effacement of haustrations, and nonspecific mucosal nodularity [11].

To date, few cases of localized intestinal amyloidosis have been reported [12, 15-40]. In 1994, Tada et al examined 30 patients with small intestinal amyloidosis. Clinical findings included malabsorption, mass lesions, diarrhea, and hematochezia. Endoscopy of the jejunum revealed polypoid lesions, wall thickening, erosions, ulcerations, and fine granular appearance. The amyloid proteins included amyloid A protein (AA), light chain protein (AL), beta 2-microglobulin, and prealbumin. Histologically, amyloid deposits were seen in the submucosa, and muscle layer [14]. Chen et al reported a case of localized amyloidosis of the transverse colon in a 52-year-old male patient. The clinical presentations included abdominal pain, bloody stool, and weight loss. Colonoscopy revealed a marked narrowing of the transverse colon associated with the presence of multiple polypoid growths. The clinical and radiological findings supported the initial impression of carcinoma, and therefore the patient underwent right hemicolectomy with lymph node dissection [15]. Grossly, there was an ulcerative mass with marked narrowing of the lumen. Histologically, amyloid deposits were seen in the lamina propria [15].

To date, our knowledge about the clinicopathological features of the localized intestinal amyloidosis is limited. To gain insights into these issues, we conducted a comprehensive literature search and meta-analysis of the relevant published literature. Also, we presented a rare case of primary isolated intestinal amyloidosis.

\section{Materials and Methods}

This is a viewpoints study (not an original research investiga- tion) that did not include any interaction or intervention with human subjects or any access to any identifiable private information. Therefore the study did not require institutional review board review [41]. This study was performed in accordance with the principles of the Declaration of Helsinki [41].

\section{Search strategy and selection criteria}

The authors adhered to Preferred Reporting Items for Systematic Reviews and Meta-Analyses (PRISMA) guidelines [41]. The literature was reviewed by searching the PubMed electronic database using the key term "amyloidosis" and the following words: "intestine" or "small intestine" or "duodenum" or "ileum" or "jejunum" or "appendix" or "colon" or "sigmoid" or "rectum" and/or "cecum" to identify the eligible studies. The search results were considered based on their titles, abstracts, and publication date. Their full texts were examined to confirm their eligibility and then were included in the study. A summary of the search strategy and selection criteria is shown in Figure $1[5,15,42,43]$.

\section{Data extraction, a methodological assessment, and statisti- cal analysis}

The clinicopathological findings were extracted including the family name of the first author, year of publication, patient age, gender, symptoms, endoscopic features, histology, special stains, and presence or absence of systemic involvement. The authors independently read and analyzed each study. They defined the final diagnosis as "the histopathological diagnosis" to ensure homogeneous evaluation of the findings. Statistical computations were performed using IBM-SPSS 21.0 (IBMSPSS Inc., Chicago, IL, USA). Descriptive statistics (mean, standard error of mean and ranges) were calculated.

\section{Results}

\section{Flow trial}

Our search yielded 756 previous studies about gastrointestinal amyloidosis, of which 513 were excluded. The remaining 243 articles have undergone "abstract review". These studies covered a period of 59 years (1960 to 2019). This relatively long period allowed proper evaluation of a good number of cases in the literature. Twenty-seven studies with the final diagnosis of localized intestinal amyloidosis were included in the present study. In all cases, the workup for monoclonal gammopathy and systemic amyloidosis (serum protein electrophoresis and pathological examination of the tissue specimens) was performed. The diagnosis of localized amyloidosis was substantiated by the use of ancillary studies including radiological examination such as abdominal ultrasound, barium enema [15], whole-body computed tomography [34], magnetic resonance imaging, positron emission tomography [28], skeletal surveys, electrocardiography and echocardiography $[12,21]$. 


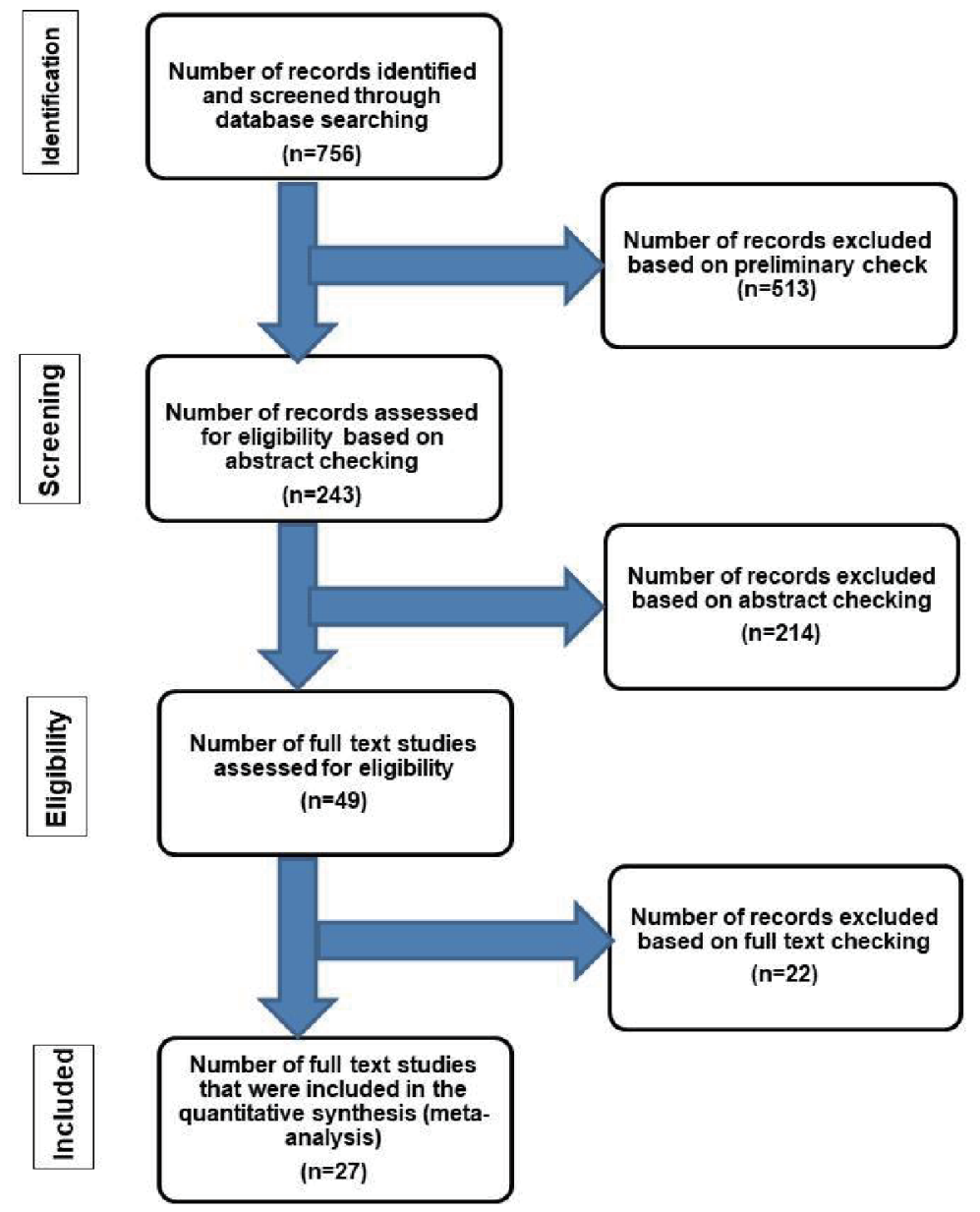

Figure 1. Flow chart of literature search and study selection for cases of localized intestinal amyloidosis. The inclusion criteria included patients who had amyloid deposition in the intestine without evidence of systemic involvement or an associated condition leading to secondary amyloidosis [42]. The exclusion criteria included patients with one or more of the followings: 1) Evidence of chronic diseases such as collagen vascular disease, that may have led to secondary amyloidosis [42]; 2) Additional organ involvement by amyloidosis; 3) Intestinal symptoms suggestive of amyloidosis but without confirmatory diagnostic tissue biopsy; 4) Plasma cell dyscrasia, myeloma, lymphoplasmacytic disorders or other B-cell malignancies such as Waldenstrom's macroglobulinemia [5]; 5) Systemic AL type [43]; 6) Laboratory findings revealing positive detection of monoclonal light chains in serum and/or urine [15].

The reported cases did not have any evidence of generalized metabolic complications or extra-intestinal complications of accumulated amyloidosis [12, 15-40]. A summary of the flow chart of the literature search and study selection criteria is shown in Figure $1[5,15,42,43]$.

\section{Clinical presentations and treatment modalities in local- ized intestinal amyloidosis}

The colorectum (44\%), jejunum (40\%), transverse colon $(11 \%)$, ileum $(7 \%)$, and duodenum $(5 \%)$ were the most commonly affected sites. The lesions were more common in males than in females. The mean age at presentation was $62 \pm 3.7$ years (small intestine) and $65.1 \pm 3.9$ years (colorectum) [12, 15-39]. In the small intestine, the main symptoms were abdominal pain $[28,33,34,36]$ and intestinal obstruction $(33 \%)$
$[29,31,35]$, followed by diarrhea and vomiting (16\%) [28, $32]$, bleeding [30, 36], malabsorption [32], and constipation [34]. In the colorectum, the main symptoms were rectal bleeding $(37 \%)[15,20-23]$, followed by abdominal pain $(11 \%)$ $[15,20]$, anemia [18], intestinal obstruction [16] and weight loss [15]. Chen et al described a case of localized amyloidosis of the transverse colon in a 52-year-old male patient who presented with a periumbilical pain, rectal bleeding, and weight loss. Colonoscopic examination revealed mucosal ulcerations and luminal narrowing by multiple polypoid lesions [15]. Treatment modalities included in these cases included drug therapy (prednisolone, azathioprine, melphalan, and colchicines) $[16,26,28]$, intestinal resection (partial jejunectomy, endoscopic submucosal resection) [21, 29], and colonic resection $[12,20]$ with close follow-up of the patients [16, 21]. A summary of these results is presented in Tables $1-3[6,12$, $15-40]$. 
Table 1. Data From Individual Studies of Primary Localized Amyloidosis of the Small Intestine

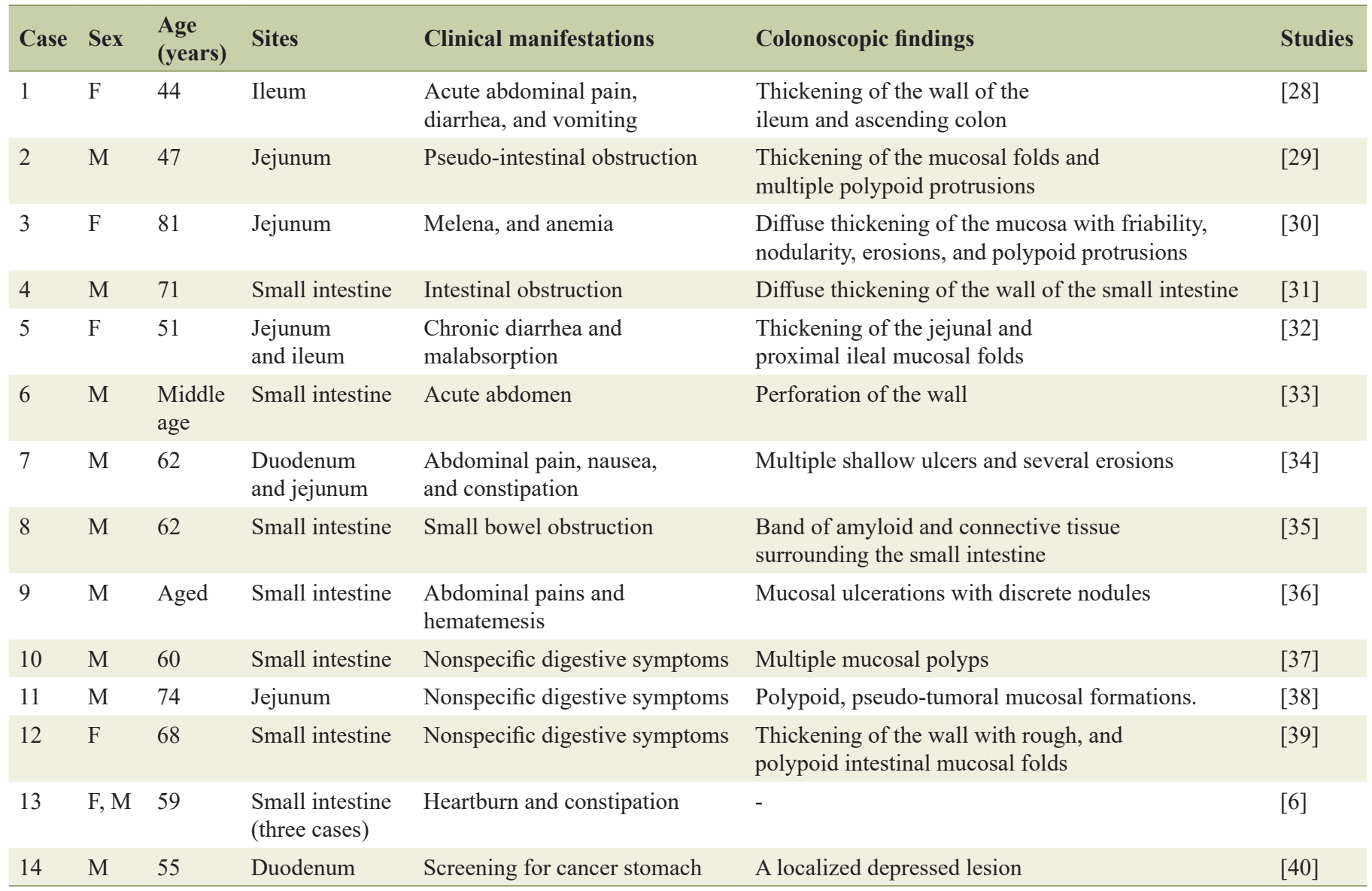

\section{Endoscopic and histological findings in localized intestinal amyloidosis}

In the small intestine, the most common endoscopic findings were thickening of the intestinal wall $(18 \%)$ [28-32, 39], and polypoid mucosal protrusions (11\%) [37-39], followed by mucosal ulceration $(7 \%)[34,36]$, friability and nodularity of the mucosa $(7 \%)[30,36]$, and wall perforations $(3 \%)[33]$. In the colorectum, polypoid masses with luminal narrowing were the most common endoscopic finding (29\%) [12, 15-18, $21]$, followed by mucosal ulcerations (solitary or multiple ulcers) $(14 \%)[20,22,25-27]$ or and thickening of the wall $(7 \%)$ $[20,27]$, nodularity and friability of the mucosa $(2 \%)[23,24$, $26]$ and perforation (3\%) [20]. In all cases, deposition of pale eosinophilic amorphous amyloid material with the prominent cracking artifact in the mucosa, submucosa, muscle layer, or walls of the blood vessels represented the main histological feature [12, 15-39]. The further Congo red staining revealed the characteristic salmon-pink color, confirming the presence of amyloid deposits. The deposits showed the characteristic apple-green birefringence under polarized light. Immunohistochemical stains were also performed in some cases to substantiate the diagnosis. Other histological features included mucosal ulceration, amyloid angiopathy, necrotizing angiitis [31], foreign body giant cell reaction [31], ischemic changes, focal active colitis, and cryptitis (a personal observation). The amyloid type was AL (light chain protein) in the majority of the cases of the small intestine $[6,16,21,23,24,34,40]$ and colorectum $[16,18,20,21,23,24]$. Other amyloid protein types included AA-amyloid [31] and high serum amyloid (SAA) in some cases [28]. Summary of clinicopathological findings in patients with localized intestinal amyloidosis is presented in Tables 1-3 [6, 12, 15-40].

\section{Case report}

\section{Primary localized amyloidosis of the intestine}

A 55-year-old female patient presented with anorexia, diarrhea, rectal bleeding, and weight loss. Colonoscopy revealed picture reminiscent of active pancolitis (friable mucosa with mucosal erosions, ulcerations, and hemorrhage) and six biopsies from the ileum, cecum, and colon (ascending, transverse, descending colons), and rectum were obtained. On histology, all biopsies showed deposition of pale eosinophilic amorphous material with the prominent cracking artifact, characteristic of amyloid, in the lamina propria and submucosa. In some areas, 
Table 2. Data From Individual Studies of Primary Localized Amyloidosis of the Colon

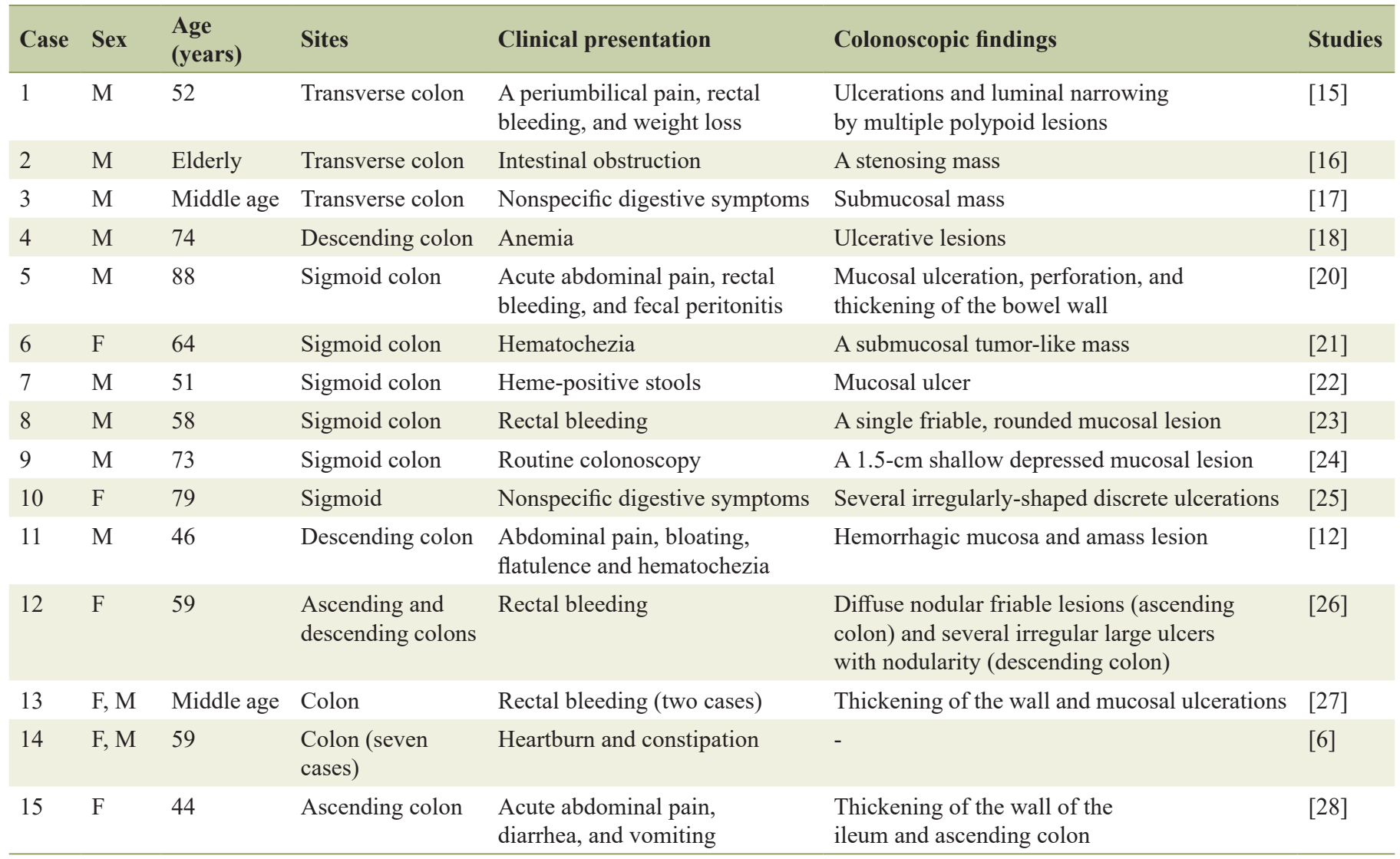

Table 3. Clinical, Colonoscopic and Histological Findings in the Primary Localized Amyloidosis of the Intestine

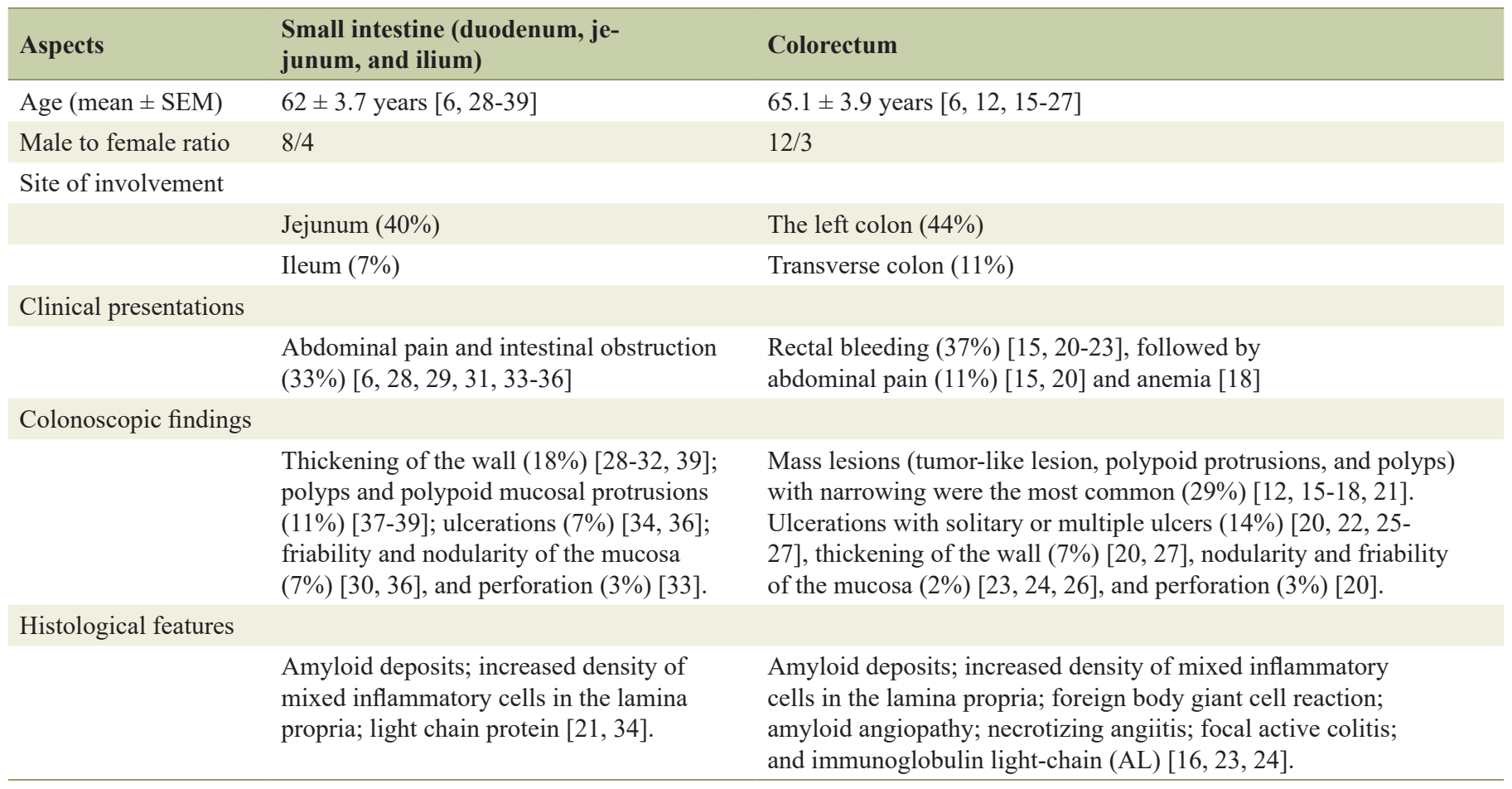



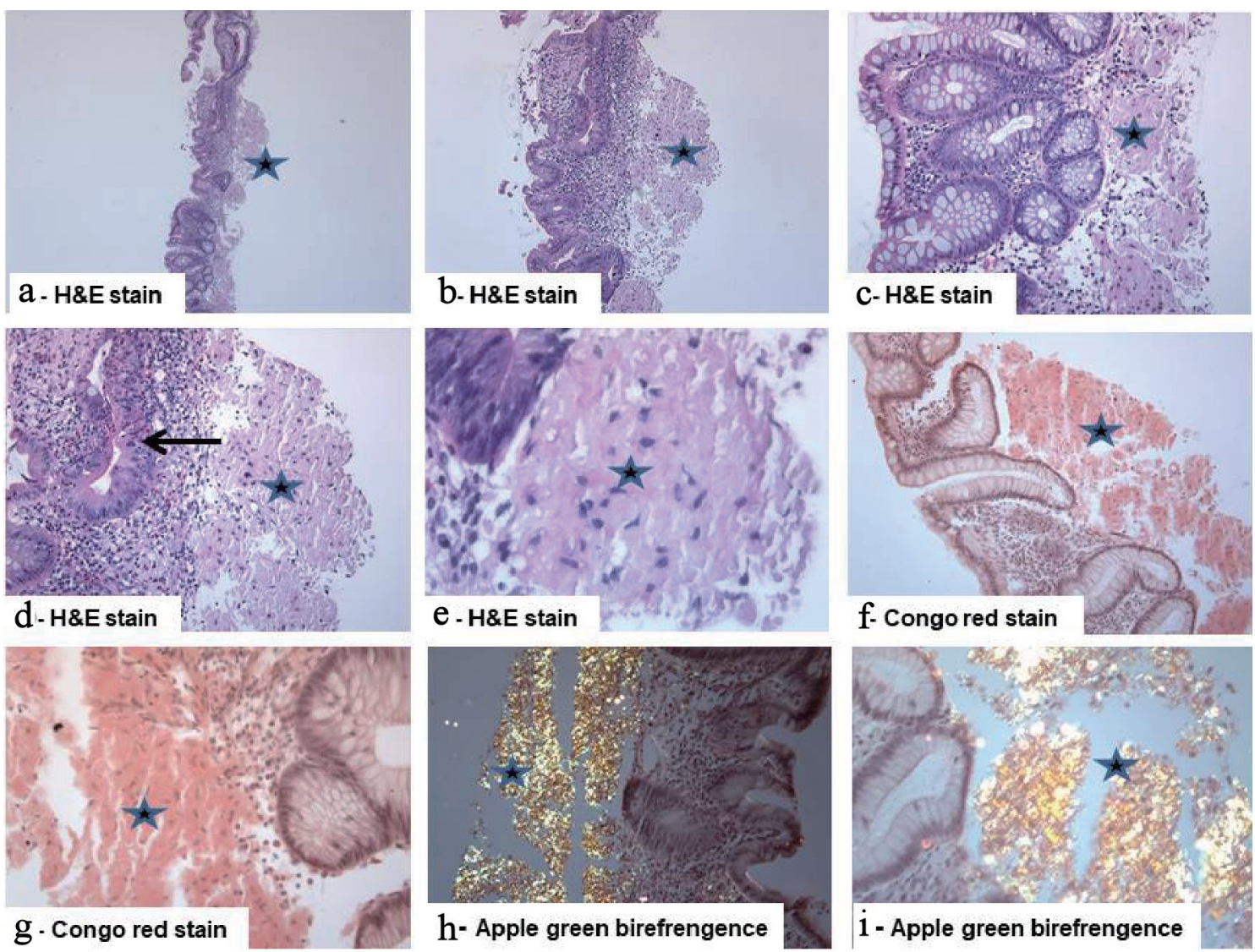

Figure 2. Isolated primary amyloidosis of the intestine (case report): (a - e) Colonic mucosa with deposition of pale eosinophilic amorphous material (star) in the submucosa with abundant deposits of pink amorphous materials with some cracking artifacts, characteristic of amyloid. Focal surface mucosal ulceration is noted. The background mucosa shows focal active inflammation, including cryptitis (arrow), and there is patchy crypt distortion. No granulomas are seen (hematoxylin and eosin: (a) $\times 20$, (b) $\times 40$, $(c, d) \times 200$, and $(e) \times 400)$. (f, g) Congo red stain which reveals the characteristic salmon-pink color, confirming the presence of amyloid deposits (Congo red, $(\mathrm{f}) \times 200$ and $(\mathrm{g}) \times 400)$. (h, i) The deposits exhibit characteristic apple-green birefringence under polarized light (Congo red under polarized light $((\mathrm{h}) \times 200$ and $(\mathrm{i}) \times 400)$.

the lamina propria was entirely replaced by these deposits. The background mucosa showed focal active inflammation (focal ulceration, cryptitis, occasional crypt abscess). This focal chronic active inflammatory change may be secondary to erosion/ulceration associated with amyloid deposits [12]. Congo red stain confirmed the presence of amyloid deposits with apple-green birefringence under polarized light (Fig. 2). Immunohistochemical analysis revealed that the amyloid deposits were positive for amyloid lambda, but negative for amyloid A. These findings were consistent with amyloid light-chain (AL) amyloidosis. We performed several laboratory and radiological investigations to rule out the possibility of systemic involvement. The hematological indices (red blood cells (RBCs) count, hemoglobin, mean corpuscular volume (MCV), mean corpuscular hemoglobin (MCH), platelets, and white blood cells count) and the biochemical tests (total protein, albumin, globulin, alkaline phosphatase, aspartate aminotransferase (AST), total bilirubin, ferritin, vitamin B12, folic acid and, prostate-specific antigen (PSA), urea, creatinine, electrolytes, and spot urine protein/creatinine ratio, protein electrophoresis and immunoelectrophoresis of serum and urine) were with- in normal limits. Thyroid function tests were within normal limits. Skeletal surveys, electrocardiograms, and radiological findings (liver, spleen, and kidneys) were unremarkable. Bone marrow biopsy showed no abnormalities. Therefore the diagnosis of localized primary intestinal AL amyloidosis involving terminal ileum, cecum, colon and rectum, associated ulceration, and inflammatory/regenerative changes was established.

The study presents a representative retrospective case in which the archival formalin-fixed paraffin-embedded tissues, from the consultation files of the authors, were used [41]. All the materials (paraffin-embedded tissue block, slides and pathology report) were coded. The information obtained was analyzed and reported in such a way that the identity of the case presented cannot absolutely be ascertained. The patient consent was obtained.

\section{Discussion}

Localized amyloidosis of the intestine is of interest to the pathologists and gastroenterologists because some cases may 
mimic tumors. Here, we present a comprehensive review of the literature about this entity. In agreement with previous reports $[12,15-40]$, our analysis indicates that the primary localized intestinal amyloidosis is a rare condition with disparate and nonspecific clinicopathological and endoscopic features. Also, its outcome depends on the site of the deposition of the amyloid (small intestine versus colorectum) [12, 15-39]. In 2013, Cowan et al examined the clinicopathological features of 2,334 patients with amyloidosis. Seventy-six patients had gastrointestinal amyloidosis. The age range of the patients was 34 - 79 years. The patients include 16 cases with localized gastrointestinal amyloidosis and 60 cases with gastrointestinal involvement as a part of systemic amyloidosis. In the latter, mutations of the transthyretin gene were found. Gastrointestinal bleeding and weight loss were the most common presenting symptoms [6].Threlkeld and Nguyen reported as a case of isolated colonic amyloidosis in a 46-year-old male patient who presented with hematochezia and abdominal pain. Colonoscopic studies revealed the presence of a tumor-like mass in the descending colon and therefore partial colectomy was performed. Grossly, the mucosa was necrotic, but no mass lesion was identified. There were light chains-positive amyloid deposits in the muscle layers, submucosa, and submucosal blood vessels. Laboratory investigations for inflammatory condition and plasma cell disorders were negative, and therefore the diagnosis of isolated gastrointestinal amyloidosis was established. The patient received colchicine with marked clinical improvement [12]. Similarly, Diaz Del Arco et al reported a case of globular amyloid deposits involving the left colon in a 74-year-old male patient presenting with anemia and colitis. Histologically, there were Congo red positive deposits in the lamina propria of the colon [18].

The duodenum is an important site for the deposition of amyloid protein. Patients with primary duodenal amyloidosis may present with epigastric pain, nausea, constipation, steatorrhea, protein loss, malabsorption, hemorrhage, and vomiting. Endoscopic findings include the thickening of the duodenal wall and the presence of polypoid lesions [29, 34, 40]. Deguchi et al described a case of an isolated duodenal and jejunal amyloidosis in a 47-year-old male patient who presented with features of intestinal pseudo-obstruction. Endoscopy revealed thickening and polypoid lesions of the duodenal mucosal folds. The further histological evaluation confirmed the presence of amyloid deposits in the duodenal and jejunal walls [29]. Similarly, Choi et al reported a case of localized amyloidosis of the small intestine (duodenum and jejunum) in a 62-year-old male patient who presented with abdominal pain, nausea, and constipation of several years duration. Endoscopy revealed mucosal erosions, ulceration, and tumor-like masses. Immunohistological analysis revealed the presence of lambda light chain protein, therefore establishing the diagnosis of amyloidosis [34].

In the primary localized amyloidosis, the isolated deposition of amyloid proteins may be due to the local tissue damage with the synthesis and deposition of amyloid substances in the individual organs [44]. The synthesis of amyloid results from plasma cell reaction to the inflammatory antigens. The otherwise benign polyclonal plasma cells produce monoclonal immunoglobulin light chains (L) that are deposited as AL amyloid [45]. It is still possible that the deposition of amyloid results from the inability of the body to clear light chains produced by plasma cells [46]. Amyloid deposits in the intestine are usually irreversible but can sometimes be reversible with chemotherapy treatment, especially with melphalan and steroid therapy [16].

In primary localized intestinal amyloidosis, we found variations in the site of deposition of amyloid proteins. There was the deposition of the amyloid proteins in the walls of the blood vessels, mucosa, submucosa, muscularis mucosae, muscularis propria, and the neural elements [47]. The destructive effects of amyloid usually resulted from the local replacement of these important histological compartments. For instance, amyloid deposition in the wall of the blood vessels usually leads to ischemia, bowel infarction [11] or intestinal perforation [48]. The involvement of the mucosa by amyloid deposits usually produces mucosal ulcerations leading to malabsorption [32], hematochezia, and anemia [12, 21]. The deposition of amyloid in the submucosa and the muscle layers usually leads to the formation of the mass lesions (amyloidoma or amyloid tumor) resulting in intestinal obstruction [16]. The involvement of the neural elements results in altered bowel motility and chronic ileus [49]. Hemorrhage (melena and bleeding per rectum) is reasoned to bowel ischemia, hemorrhagic necrosis of the mucosa, damage of the vessel walls, and abnormal platelet aggregation [30].

The variations in the gross findings of intestinal amyloidosis may be reasoned to the type and the site of deposition of the amyloid protein. When the deposition of the amyloid protein occurs predominantly in the perivascular and interstitial spaces, it usually produces diffuse thickening of the intestinal wall rather than polypoid or tumor-like lesions [20, 27, 28, 31, 32]. Elevations or localized tumor-like lesions may reflect the presence of nodular aggregates of plasma cells locally producing abundant amyloid proteins, which usually originate from AL protein produced by local aggregates of plasma cells involving the mucosa, submucosa or the muscle layer $[15,17,21,30$, 37]. Fine granular lesions or mucosal erosion or ulcerations are commonly associated with the deposition of AA amyloidosis $[18,20,34]$.

To conclude, the data presented here provides an in-depth characterization of the clinicopathological features of primary localized intestinal amyloidosis. It indicates that this entity is rare and its presenting features are generally nonspecific and therefore the diagnosis is challenging and can be easily missed. Knowledge of this type of amyloidosis is warranted to avoid aggressive management such as treatment with an alkylating agent. A high index of suspicion should be maintained both on the part of the gastroenterologists and the histopathologists.

\section{Acknowledgments}

None to declare.

\section{Financial Disclosure}

None to declare. 


\section{Conflict of Interest}

None to declare.

\section{Informed Consent}

Informed consent was obtained.

\section{Author Contributions}

Saeed Ali Alshehri conceived and designed the analyses, collected the data (biochemical and clinicopathologic aspects of amyloidosis), performed the analyses tools including the statistical data analysis, and wrote the paper. Mahmoud Rezk Abdelwahed Hussein supervised the data analysis and contributed to the conception of the idea of the manuscript, data collection (Histological aspects of amyloidosis), writing up and revising of the manuscript.

\section{Data Availability}

The authors declare that data supporting the findings of this study are available within the article.

\section{References}

1. Westermark P, Benson MD, Buxbaum JN, Cohen AS, Frangione B, Ikeda S, Masters CL, et al. Amyloid fibril protein nomenclature - 2002. Amyloid. 2002;9(3):197200.

2. Monge M, Chauveau D, Cordonnier C, Noel LH, Presne C, Makdassi R, Jaureguy M, et al. Localized amyloidosis of the genitourinary tract: report of 5 new cases and review of the literature. Medicine (Baltimore). 2011;90(3):212222.

3. Mathis H. [Rokitansky, Virchow and Heschl on the problem of amyloidosis. (a historical study)]. Zentralbl Allg Pathol. 1968;111(2):103-107.

4. Sack GH, Jr. Serum amyloid A - a review. Mol Med. 2018;24(1):46.

5. Gillmore JD, Wechalekar A, Bird J, Cavenagh J, Hawkins S, Kazmi M, Lachmann HJ, et al. Guidelines on the diagnosis and investigation of AL amyloidosis. Br J Haematol. 2015;168(2):207-218.

6. Cowan AJ, Skinner M, Seldin DC, Berk JL, Lichtenstein DR, O'Hara CJ, Doros G, et al. Amyloidosis of the gastrointestinal tract: a 13-year, single-center, referral experience. Haematologica. 2013;98(1):141-146.

7. Ankarcrona M, Winblad B, Monteiro C, Fearns C, Powers ET, Johansson J, Westermark GT, et al. Current and future treatment of amyloid diseases. J Intern Med. 2016;280(2):177-202.

8. Selkoe DJ. Folding proteins in fatal ways. Nature. 2003;426(6968):900-904.
9. Otaka Y, Goda F, Nakazato Y, Tsutsui T. Systemic heavyand light-chain amyloidosis presenting nephrotic syndrome and congestive heart failure: a case presentation and literature review. Amyloid. 2019;26(sup1):95-96.

10. Picken MM. The pathology of amyloidosis in classification: a review. Acta Haematol. 2020:1-13.

11. Trinh TD, Jones B, Fishman EK. Amyloidosis of the colon presenting as ischemic colitis: a case report and review of the literature. Gastrointest Radiol. 1991;16(2):133-136.

12. Threlkeld C, Nguyen TH. Isolated amyloidosis of the colon. J Am Osteopath Assoc. 1996;96(3):188-190.

13. Garcia-Gonzalez R, Fernandez FA, Garijo MF, Fernando Val-Bernal J. Amyloidosis of the rectum mimicking collagenous colitis. Pathol Res Pract. 1998;194(10):731735.

14. Tada S, Iida M, Yao T, Kawakubo K, Yao T, Okada M, Fujishima M. Endoscopic features in amyloidosis of the small intestine: clinical and morphologic differences between chemical types of amyloid protein. Gastrointest Endosc. 1994;40(1):45-50.

15. Chen JH, Lai SJ, Tsai PP, Chen YF. Localized amyloidosis mimicking carcinoma of the colon. AJR Am J Roentgenol. 2002;179(2):536-537.

16. Racanelli V, D'Amore FP. Localized AL amyloidosis of the colon and clinical features of intestinal obstruction. A case report. Ann Ital Med Int. 1999;14(1):58-60.

17. Watanabe T, Kato K, Sugitani M, Kaneda N, Hoshino N, Imatake $\mathrm{K}$, Matsui $\mathrm{T}$, et al. A case of solitary amyloidosis localized within the transverse colon presenting as a submucosal tumor. Gastrointest Endosc. 1999;49(5):644647.

18. Diaz Del Arco C, Fernandez Acenero MJ. Globular amyloidosis of the colon. Arab J Gastroenterol. 2018;19(2):96-99.

19. Tokoro C, Inamori M, Sekino Y, Sakai E, Uchiyama T, Iida $\mathrm{H}$, Hosono $\mathrm{K}$, et al. Localized primary AL amyloidosis of the colon without other GI involvement. Gastrointest Endosc. 2011;74(4):925-926; discussion 927.

20. Parks RW, O'Rourke D, Bharucha H, Wilson BG. Perforation of the sigmoid colon secondary to localised amyloidosis. Ulster Med J. 2002;71(2):144-146.

21. Ogasawara N, Kitagawa W, Obayashi K, Itoh Y, Noda H, Funaki Y, Yokoi T, et al. Solitary amyloidosis of the sigmoid colon featuring submucosal tumor caused hematochezia. Intern Med. 2013;52(22):2523-2527.

22. Hirata K, Sasaguri T, Kunoh M, Shibao K, Nagata N, Itoh H. Solitary "amyloid ulcer" localized in the sigmoid colon without evidence of systemic amyloidosis. Am J Gastroenterol. 1997;92(2):356-357.

23. Martin-Arranz E, Pascual-Turrion JM, Martin-Arranz MD, Burgos E, Froilan-Torres C, Adan-Merino L, Lorenzo A, et al. Focal globular amyloidosis of the colon. An exceptional diagnosis. Rev Esp Enferm Dig. 2010;102(9):555-556.

24. Makazu M, Nakajima T. A case of localized AL amyloidosis of the sigmoid colon with lymphocytes exhibiting a premalignant status. Jpn J Clin Oncol. 2013;43(7):767.

25. Matsui H, Kato T, Inoue G, Onji M. Amyloidosis localized in the sigmoid colon. J Gastroenterol. 1996;31(4):607- 
611.

26. Kwon YH, Kim JY, Kim JH, Park HW, Yang HM, Jeon SW, Kim SK. [A case of primary colon amyloidosis presenting as hematochezia]. Korean J Gastroenterol. 2012;59(1):44-47.

27. Kyle RA, Gertz MA, Lacy MQ, Dispenzieri A. Localized AL amyloidosis of the colon: an unrecognized entity. Amyloid. 2003;10(1):36-41.

28. Kim YJ, Kim HS, Park SY, Park SW, Choi YD, Park CH, Choi SK, et al. Intestinal amyloidosis with intractable diarrhea and intestinal pseudo-obstruction. Korean J Gastroenterol. 2012;60(3):172-176.

29. Deguchi M, Shiraki K, Okano H, Yamanaka T, Ito N, Takase K, Nakano T. Primary localized amyloidosis of the small intestine presenting as an intestinal pseudo-obstruction: report of a case. Surg Today. 2001;31(12):10911093.

30. Yau AH, Cornell IS, Cheung J. Jejunal amyloidosis: a rare cause of severe gastrointestinal bleeding. Can J Gastroenterol. 2012;26(6):320-321.

31. Oweity T, West AB, Stokes MB. Necrotizing angiitis of the small intestine related to AA-amyloidosis: a novel association. Int J Surg Pathol. 2001;9(2):149-154.

32. Harish K, Gokulan C. Selective amyloidosis of the small intestine presenting as malabsorption syndrome. Trop Gastroenterol. 2008;29(1):37-39.

33. Griffel B, Man B, Kraus L. Selective massive amyloidosis of small intestine. Arch Surg. 1975;110(2):215-217.

34. Choi JH, Ko BM, Kim C, Kim HK, Han JP, Hong SJ, Moon JH, et al. A case of localized amyloid lightchain amyloidosis in the small intestine. Intest Res. 2014;12(3):245-250.

35. Jones J, VanRosendaal G, Cleary C, Urbanski S, Woodman RC. Primary amyloidosis presenting as small bowel encapsulation. Can J Gastroenterol. 2004;18(3):169-172.

36. Pandarinath GS, Levine SM, Sorokin JJ, Jacoby JH. Selective massive amyloidosis of the small intestine mimicking multiple tumors. Radiology. 1978;129(3):609-610.

37. Shimizu S, Yoshinaka M, Tada M, Kawamoto K, Inokuchi $\mathrm{H}$, Kawai K. A case of primary amyloidosis confined to the small intestine. Gastroenterol Jpn. 1986;21(5):513517.
38. Peny MO, Debongnie JC, Haot J, Van Gossum A. Localized amyloid tumor in small bowel. Dig Dis Sci. 2000;45(9):1850-1853.

39. Grigor'ev BA, Suslonov AN. [Local tumoral amyloidosis of the small intestine]. Arkh Patol. 1989;51(3):70-73.

40. Akamatsu T, Watanabe N, Chiba T. Primary localized amyloidosis of the duodenum. Clin Gastroenterol Hepatol. 2007;5(2):e4-5.

41. Moher D, Liberati A, Tetzlaff J, Altman DG, The PRISMA Group. Preferred reporting items for systematic reviews and meta-analyses: the PRISMA statement. J Clin Epidemiol. 2009;62(10):1006-1012.

42. Levy A, Lender M. [Gastrointestinal amyloidosis]. Nouv Presse Med. 1976;5(31):1969-1973.

43. Fukui T, Tanimura Y, Matsumoto Y, Horitani S, Tomiyama T, Okazaki K. Incidentally Detected Amyloid LightChain Amyloidosis Caused by Monoclonal Gammopathy of Undetermined Significance: Possible Time-Dependent Change in Colonic Findings. Case Rep Gastroenterol. 2018;12(3):737-746.

44. Westermark P. Localized AL amyloidosis: a suicidal neoplasm? Ups J Med Sci. 2012;117(2):244-250.

45. Mollee P, Renaut P, Gottlieb D, Goodman H. How to diagnose amyloidosis. Intern Med J. 2014;44(1):7-17.

46. Berg AM, Troxler RF, Grillone G, Kasznica J, Kane K, Cohen AS, Skinner M. Localized amyloidosis of the larynx: evidence for light chain composition. Ann Otol Rhinol Laryngol. 1993;102(11):884-889.

47. Lim AY, Lee JH, Jung KS, Gwag HB, Kim DH, Kim SJ, Lee GY, et al. Clinical features and outcomes of systemic amyloidosis with gastrointestinal involvement: a singlecenter experience. Korean J Intern Med. 2015;30(4):496505.

48. Ishizaki Y, Nobori M, Tanaka N, Kanamori Y, Asada M, Saiki S. Perforation and tumor formation of the intestine in primary amyloidosis. Am J Gastroenterol. 1991;86(3):363-366.

49. Janot MS, Kersting S, Chromik AM, Tannapfel A, Uhl W. [Amyloidosis of the small intestine following whipple operation is a rare cause of chronic ileus and has to be considered as differential diagnosis]. Zentralbl Chir. 2010;135(4):345-349. 